\title{
Relationship between an atomic decomposition of double and unary systems in grand-Lebesgue spaces
}

\author{
FATIMA GULIYEVA \\ Institute of Mathematics and Mechanics of NAS of Azerbaijan, \\ AZ1141, 9, B.Vahabzade Str., \\ Baku \\ AZERBAIJAN
}

\begin{abstract}
The grand-Lebesgue space is defined. Based on the shift operator, a separable subspace is determined in which continuous functions are dense. The concepts of frame and atomic decomposition are defined. An atomic decomposition of double and unary systems of functions in grand-Lebesgue spaces is considered. Relationship between atomic decomposition of these systems in grand-Lebesgue spaces is established
\end{abstract}

Key-Words: Atomic decomposition, grand-Lebesgue spaces, double and unary systems, frame

Received: December 15, 2019. Revised: March 7, 2020. Accepted: March 20, 2020. Published: March 31, 2020.

\section{Introduction}

Frame theory has a diverse array of applications in many scientific fields, like physics, chemistry, biology, medicine, data compression, signal processing, and others that's why the global interest in it is growing at a rapid pace. Many monographs and review articles of different mathematicians have been dedicated to this theory. More detailed can be found in monographs by R.Young [1], Daubechies I. [2], O.Christensen [3-6], Chui Ch. [7], a review article of Dremin I.M., Ivanov O.V., Nechitailo V.A. [8], etc. This theory dates back to the seminal paper by R.J.Duffin and A.C.Schaeffer [9]. Later, there appeared various generalizations of the concept of frame. Subsequently, it has evolved in different directions. Naturally is scientifically interesting methods of acquisition of a frame. There are various methods of education frames. One of these methods is a perturbation method. Many results have been obtained using this method in the context of classical Paley-Wiener theorem on the Riesz basicity of perturbed system of exponents , the results on sustainability properties of the frame are obtained in a series of papers by O.Christensen (respect to these results can be found in [3-6] ).

In the context of applications to various branches of mathematics, for example, such as theory of partial differential equations, theory of approximations, harmonic analysis, etc., there arose great interest in nonclassical function spaces. As examples of such spaces, we can mention Lebesgue space with variable summability index, Morrey space, grand Lebesgue space, etc. A lot of articles, reviews and monographs have been dedicated to these spaces (see, e.g., F. Xianling, Z. Dun [22], I.I.
Sharapudinov [23], C.T. Zorko [24], C.B. Morrey [25], D.V. Cruz-Uribe, Fiorenza A. [26], Adams [27], S. Samko [28], Kokilashvili [29], R.E.Castilo, H.Rafeiro [30], H. A. Fiorenza, G. E. Karadzhov [31], B.T. Bilalov, T.B.Gasymov, A.A. Guliyeva [32] etc.). Along with this, of course, one has to study approximation matters in suchlike spaces. Approximation matters have been (and are being) relatively well studied in generalized Lebesgue spaces by I.I. Sharapudinov [33], D. Israfilov, Tozman N. P. [34, 35], Bilalov B. T., Guseynov Z.G. [36], etc. The situation is different with the case of Morrey-type and grand Lebesgue spaces, and only recently the approximation matters began to be studied in these spaces. Many problems in this field still remain to be solved. Apparently the works by D. Israfilov, Tozman N. P. [34, 35], B.T. Bilalov, A.A. Guliyeva [37], have been pioneers in this field.

Note that the grand-Lebesgue spaces $L^{p)}$ first is appeared in the paper [38], where the integrability problem of the Jacobian was treated under a minimal hypothesis. In particular, it is shown that if $f=\left(f_{1}, \ldots, f_{n}\right): \Omega \rightarrow R^{n}$, where $\Omega$ is an open subset in $R^{n}, n \geq 2$, then the Jacobian determinant of $f$ belongs to the class $L_{l o c}^{\prime}(\Omega)$ provided that $g \in L^{n)}$, where

$$
g(x):=\left\{\sup D f(x) y: y \in S^{n-1}\right\},
$$

where $S^{n-1}$ is a unit boll in $R^{n-1}$.

Feichtinger and Grochenig (see e.g. [18-20]) proved that the group-theoretic setup even allows us to obtain series expansions in a large scale of Banach spaces, a result which leads Grochenig to 
define frames in Banach spaces. By removing some of the conditions we obtain $p$-frames, first studied separately by Aldroubi, Sun and Tang [21].

So, this paper is organized as follows. It consists of three sections. The first section is an introduction. This section provides a brief history of the emergence of frame theory. Recently, there has been an increase in interest in studying various problems of mathematics in non-standard function spaces, and the relevance of the topics discussed in the article is substantiated. The second partsection is devoted to the necessary concepts and facts that we use in this paper. The grand-Lebesgue space $L^{p)}(a, b)$ is defined. This space is non-separable. Based on the shift operator, a separable subspace $G^{p)}(a, b) \subset L^{p)}(a, b)$ is determined in which continuous functions are dense. The concepts of frame and atomic decomposition are defined. The third section is devoted to the main results. It considers a unary system of a general form and, following this system, a double system is determined. It is proved that if a unary system is an atomic decomposition of $G^{p)}(0, a)$, then a double system is also an atomic decomposition of $G^{p)}(-a, a)$. It should be noted that similar relationships were previously established with respect to the basis properties (completeness, minimality, basicity ) of power systems in [10-15].

\section{Needful Information}

We will use the standard notation. $N$ will be a set of all positive integers; $Z$ will be a set of all integers; $Z_{+} \equiv\{0\} \cup N ; C$ will be the set of complex numbers; Banach space will be referred to as $B$-space; $X^{*}$ will stand for a space conjugated to $X ; \quad\|\cdot\|_{X}$ will denote a norm in the space $X ; K$ is a field of scalars; $\delta_{n k}$ will be the Kronecker symbol; Throughout this paper $\vec{y}$ will denote $\vec{y} \equiv\left\{y_{n}\right\}_{n \in N}$. mesM will denote the Lebesgue measure of the set $M$. In the sequel, we will assume that $\mathscr{K}$ is some $B$ - space of sequence of scalars. Such space will be called $K$-space.

Let us give definitions for grand Lebesgue spaces. We also state some of their properties and auxiliary facts to be used later. By $L^{p)}(a ; b), 1<p<+\infty$, we denote a grand
Lebesgue space of measurable functions $f$ on $[a ; b]$ with the norm

$$
\|f\|_{L^{p}(a, b)}=\sup _{0<\varepsilon<p-1}\left(\frac{\varepsilon}{b-a} \int_{a}^{b}|f(t)|^{p-\varepsilon} d t\right)^{\frac{1}{p-\varepsilon}}<+\infty .
$$

The following inclusions hold

$$
\begin{aligned}
L^{p}(a ; b) & \subset L^{p)}(a ; b) \subset L^{p-\varepsilon}(a ; b), \\
1 & <p<+\infty .
\end{aligned}
$$

Obviously, the space of infinitely differentiable functions $C^{\infty}[a ; b]$ is embedded in $L^{p)}(a ; b)$. The space $L^{p)}(a ; b)$ with the norm $\|f\|_{L^{p}(a, b)}$ is a non-separable Banach space. The space $C_{0}^{\infty}[a ; b]$ of infinitely differentiable finite functions on $[a ; b]$ is not dense in $L^{p)}(a ; b)$. The validity of this assertion follows from the statement below.

Statement 2.1 [9]. The subspace $\overline{C_{0}^{\infty}[a ; b]}$ consists of the functions $f \in L^{p)}(a ; b)$ which satisfy the condition

$$
\lim _{\varepsilon \rightarrow+0} \varepsilon \int_{a}^{b}|f(t)|^{p-\varepsilon} d t=0,
$$

where $\overline{C_{0}^{\infty}[a ; b]}$ is a closure of $C_{0}^{\infty}[a ; b]$ in $L^{p)}(a ; b)$.

Extending every function $f \in L^{p)}(a ; b)$ to the whole axis $R$ and assuming $f(t)=0$, $t \in R \backslash[a ; b]$, consider the set $\widetilde{G}^{p)}(a ; b)$ of functions $f \in L^{p)}(a ; b)$ which satisfy the condition

$$
\|f(\cdot+\delta)-f(\cdot)\|_{L^{p)}(a, b)} \rightarrow 0, \delta \rightarrow 0 .
$$

It is clear that $\widetilde{G}^{p)}(a ; b)$ is a linear manifold in $L^{p)}(a ; b)$. Let $G^{p)}(a ; b)$ be its closure in $L^{p)}(a ; b)$. The space $C_{0}^{\infty}[a ; b]$ is dense in $G^{p)}(a ; b)$. Every function $f \in G^{p)}(a ; b)$ satisfies (2.1). In fact, it is easy to show that (2.1) is true for every function $f \in C_{0}^{\infty}[a ; b]$, and therefore, it is true for every function $f \in G^{p)}(a ; b)$.

Let's recall some concepts and facts from the theory of frames. First, let us give a definition of atomic decomposition. 
Definition 2.1. Let $X$ be a B-space and be a $K$-space. Let $\left\{f_{k}\right\}_{k \in N} \subset X$, $\left\{g_{k}\right\}_{k \in N} \subset X^{*}$. Then $\left(\left\{g_{k}\right\}_{k \in N},\left\{f_{k}\right\}_{k \in N}\right)$ is an atomic decomposition of $X$ with respect to $\mathscr{K}$ if :

(i) $\left\{g_{k}(f)\right\}_{k \in N} \in \mathrm{K}, \forall f \in X$;

(ii) $\exists A, B>0$ :

$A\|f\|_{X} \leq\left\|\left\{g_{k}(f)\right\}_{k \in N}\right\|_{\mathrm{K}} \leq B\|f\|_{X}, \quad \forall f \in X ;$

(iii) $f=\sum_{k=1}^{\infty} g_{k}(f) f_{k}, \forall f \in X$.

Accept the definition of $\mathscr{K}$ - Besselian.

Let $X$ be $B$-space on the field of $K$ and $\left\{x_{n}\right\}_{n \in N} \subset X$ be a minimal system with a conjugate $\left\{x_{n}^{*}\right\}_{n \in N} \subset X^{*}$. Let $\mathscr{K}$ be some $K$ space.

We will say that the system $\left\{x_{n}\right\}_{n \in N}$ has the $\mathscr{K}$-Besselian, if $\left\{x_{n}^{*}(x)\right\}_{n \in N} \in \mathscr{K}, \forall x \in X$.

About these and other facts one can see the works $[16 ; 17]$.

The concept of frame is a generalization of the concept of atomic decomposition.

Definition 2.2. Let $X$ be a B-space and $\mathscr{K}$ be a $K$-space. Let $\left\{g_{k}\right\}_{k \in N} \subset X^{*}$, and $S: \mathscr{K} \rightarrow X$ be some bounded operator. Then $\left(\left\{g_{k}\right\}_{k \in N}, S\right)$ forms a Banach frame for $X$ with respect to $\mathscr{K}$ if :

(i) $\left\{g_{k}(f)\right\}_{k \in N} \in \mathscr{L}, \forall f \in X$;

(ii) $\exists A, B>0$ :

$A\|f\|_{X} \leq\left\|\left\{g_{k}(f)\right\}_{k \in N}\right\|_{\mathrm{K}} \leq B\|f\|_{X}, \quad \forall f \in X ;$

(iii) $S\left[\left\{g_{k}(f)\right\}_{k \in N}\right]=f, \forall f \in X$.

$A$ and $B$ will be called frame bounds.

The following statement is true.

Statement 2.2 [5]. Let $X$ be a B-space and $\mathscr{K}$ be a $K$-space with a canonical basis $\left\{\delta_{n}\right\}_{n \in N}$, where $\delta_{n} \equiv\left\{\delta_{k n}\right\}_{k \in N}$. Let $\left\{g_{k}\right\}_{k \in N} \subset X^{*}$ and $S \in L(\mathscr{K} X)$. Then the following statements are equivalent to each other:

(i) $\left(\left\{g_{k}\right\}_{k \in N}, S\right)$ forms a Banach frame for $X$ with respect to $\mathscr{K}$.

(ii) $\quad\left(\left\{g_{k}\right\}_{k \in N},\left\{S\left(\delta_{k}\right)\right\}_{k \in N}\right)$ is an atomic decomposition of $X$ with respect to $\mathscr{K}$.
Let $X$ be $B$-space, $\mathscr{K}$ be some $K$ space and $\left(\vec{x}^{+} ; \vec{x}^{-}\right) \subset X$ be some double system, where $\vec{x}^{ \pm} \equiv\left\{x_{n}^{ \pm}\right\}_{n \in N}$. Let $\left(\vec{\vartheta}^{+} ; \vec{\vartheta}^{-}\right) \subset X^{*}$. Under an atomic decomposition $\left\{\left(\vec{\vartheta}^{+} ; \vec{\vartheta}^{-}\right),\left(\vec{x}^{+} ; \vec{x}^{-}\right)\right\}$of $X$ with respect to $\mathscr{K}$ we will mean the followinge:

i) The following expansion holds

$$
x=\sum_{n=1}^{\infty}\left(\vartheta_{n}^{+}(x) x_{n}^{+}+\vartheta_{n}^{-}(x) x_{n}^{-}\right), \forall x \in X ;
$$

ii) $\exists A ; B>0$ :

$A\|x\|_{X} \leq\left\|\left\{\vartheta_{n}^{+}(x)\right\}_{n \in N}\right\|_{\mathrm{K}}+\left\|\left\{\vartheta_{n}^{-}(x)\right\}_{n \in N}\right\|_{\mathrm{K}} \leq\|x\|_{X}$.

We will also need the following

Lemma 2.1. Let $f \in G^{p)}(a, b), \quad 1<p<$ $+\infty$, be an arbitrary function. Then $\left\|f \chi_{E}\right\|_{p)} \rightarrow 0$, as $|E| \rightarrow 0$, where $E \subset(a, b)$ is an arbitrary interval, $|E|$ is the length of this interval.

Indeed, let $f \in G^{p)}(a, b)$ be an arbitrary function and $\delta>0$ be an arbitrary number. Since, $C[a, b]$ is dense in $G^{p)}(a, b)$, then it is clear that $\exists g \in C[a, b]:\|f-g\|_{L^{p}(a, b)}<\delta$. If $|E|$ is sufficiently small we have

$\left\|f \chi_{E}\right\|_{L^{p)}} \leq\left\|(f-g) \chi_{E}\right\|_{L^{p)}}+\left\|g \chi_{E}\right\|_{L^{p}} \leq\|f-g\|_{L^{p}}+$ $\|g\|_{\infty}\left\|\chi_{E}\right\|_{L^{p)}}<\delta+\|g\|_{\infty} \sup _{0<\varepsilon<p-1}\left(\frac{\varepsilon}{2 \pi}\right)^{\frac{1}{p-\varepsilon}}|E| \frac{1}{p-\varepsilon}<2 \delta$.

From the arbitrariness of $\delta$ we obtain what is required.

We define the space $\left(G^{p)}(a, b)\right)^{\prime}$ associated with $G^{p)}(a, b)$ and briefly denote it by $G^{\prime}$. Let $S$ be unit ball in $G^{p)}(a, b)$, i.e.

$$
S=\left\{f \in G^{p)}(a, b):\|f\|_{p)} \leq 1\right\} .
$$

$G^{\prime}$ is a Banach space of measurable functions on $(a, b)$ for which the norm

$$
\|g\|_{G^{\prime}}=\sup _{f \in S}\left|\int_{a}^{b} f g d t\right|<+\infty
$$

is finite.

Now we state the following known (see, for example, [19])

Proposition 2.1[19]. The conjugate space $X^{*}$ to a Banach space of functions $X$ is isometrically isomorphic to the associated 
space $X^{\prime}$ if and only if $X$ has an absolutely continuous norm.

Taking into account Lemma 2.1, in particular, from this statement we obtain that $M^{\prime}$ is isometrically isomorphic to the conjugate to the space $G^{p)}(a, b)$ for $1<p<+\infty$, and denote it by $M^{*}=\left(G^{p)}(a, b)\right)^{*}$.

We will also need the following completeness criterion of a system in a Banach space $X$.

Proposition 2.2. System $\left\{x_{n}\right\}_{n \in N} \subset X$ in Banach space $X$ is complete if and if only the zero functional cancels it, i.e. from the relation $v \in X^{*}: v\left(x_{n}\right)=0, n \in N$, it follows that $v=0$, where $v(x)$ is value of functional $v \in X^{*}$ at the point $x$.

\section{Main results}

Let us consider the unary system of the form

$$
x_{n}^{ \pm}(t) \equiv \varphi_{n}(t) \pm \psi_{n}(t), \quad n \in N,
$$

where $\varphi_{n} ; \psi_{n}:[0, a] \rightarrow C$ are complex-valued functions. Using the functions $\varphi_{n}$ and $\psi_{n}$ will determine the new system on a segment $[-a, a]$. Then we will establish the connection between an atomic decomposition and frameness of these systems.

So, let us form the new system

$$
\Phi_{n}(t) \equiv\left\{\begin{array}{l}
\varphi_{n}(t), t \in[0, a], \\
\psi_{n}(-t), t \in[-a, 0),
\end{array}\right.
$$

and put

$$
\Psi_{n}(t)=\Phi_{n}(-t), \forall t \in[-a, a] .
$$

Let $\left\{\vartheta_{n}^{ \pm}\right\} \subset L_{q}(0, a)$ be some system. Similarly we define

$$
\Omega_{k}^{ \pm}(t) \equiv\left\{\begin{array}{l}
\vartheta_{k}^{ \pm}(t), t \in(0, a), \\
\pm \vartheta_{k}^{ \pm}(-t), t \in(-a, 0),
\end{array}\right.
$$

and let

$$
h_{k}^{ \pm}(t) \equiv \frac{1}{2}\left[\Omega_{k}^{+}(t) \pm \Omega_{k}^{-}(t)\right], \forall t \in(-a, a) .
$$

It is easy to see that the following relations are valid which will be used in the obtaining of the main results

$$
h_{k}^{+}(-t)=h_{k}^{-}(t), h_{k}^{-}(-t)=h_{k}^{+}(t), \forall t \in(-a, a) .
$$

We will consider the double system $\{\vec{\Phi} ; \vec{\Psi}\}$ and establish connections between the atomic decomposition of this system and unary systems $\left\{\vec{x}^{ \pm}\right\}$in grand-Lebesgue spaces $G^{p)}$.

The following theorem is true.

Theorem 3.1. Let $\left(\vec{\vartheta}^{+} ; \vec{x}^{+}\right)$and $\left(\vec{\vartheta}^{-} ; \vec{x}^{-}\right)$ be atomic decompositions of $G^{p)}(0, a)$ with respect to some $K$-space $\mathscr{K}$. Then $\left(\left(\vec{h}^{+} ; \vec{h}^{-}\right),(\vec{\Phi} ; \vec{\Psi})\right)$ is an atomic decomposition of $G^{p)}(-a, a)$ with respect to $\mathscr{K}$, where $\vec{h}^{ \pm}$determined by (3.1).

Proof. Take $\forall f \in G^{p)}(-a, a)$. Assume

$$
\left[S_{m} f\right](x)=
$$

$\sum_{n=1}^{m}\left[\int_{-a}^{a} f(t) \bar{h}_{n}^{+}(t) d t \Phi_{n}(x)+\int_{-a}^{a} f(t) \bar{h}_{n}^{-}(t) d t \Psi_{n}(x)\right]$,

$\forall m \in N$.

We have

$$
\begin{gathered}
I_{m}^{(-a, a)}=\left\|\left[S_{m} f\right](x)-f(x)\right\|_{L^{p)}(-a, a)}= \\
=\| \sum_{n=1}^{m} \frac{1}{2}\left[\int_{-a}^{a} f(t) \bar{\Omega}_{n}^{+}(t) d t \Phi_{n}(x)+\int_{-a}^{a} f(t) \bar{\Omega}_{n}^{-}(t) d t \Phi_{n}(x)+\right. \\
\int_{-a}^{a} f(t) \bar{\Omega}_{n}^{+}(t) d t \Phi_{n}(-x)- \\
\left.-\int_{-a}^{a} f(t) \bar{\Omega}_{n}^{-}(t) d t \Phi_{n}(-x)\right]-f(x) \|_{L_{(-a, a)}} .
\end{gathered}
$$

Assume the following notation

$$
\begin{aligned}
& I_{n}^{(1)}=\int_{-a}^{a} f(t) \bar{\Omega}_{n}^{+}(t) d t \Phi_{n}(x)=\int_{0}^{a}[f(t)+f(-t)] \bar{\vartheta}_{n}^{+}(t) d t \Phi_{n}(x), \\
& I_{n}^{(2)}=\int_{-a}^{a} f(t) \bar{\Omega}_{n}^{-}(t) d t \Phi_{n}(x)=\int_{0}^{a}[f(t)-f(-t)] \bar{\vartheta}_{n}^{-}(t) d t \Phi_{n}(x), \\
& I_{n}^{(3)}=\int_{-a}^{a} f(t) \bar{\Omega}_{n}^{+}(t) d t \Phi_{n}(-x)=\int_{0}^{a}[f(t)+f(-t)] \bar{\vartheta}_{n}^{+}(t) d t \Phi_{n}(-x), \\
& I_{n}^{(4)}=\int_{-a}^{a} f(t) \bar{\Omega}_{n}^{-}(t) d t \Phi_{n}(-x)=\int_{0}^{a}[f(t)-f(-t)] \bar{\vartheta}_{n}^{-}(t) d t \Phi_{n}(-x) .
\end{aligned}
$$

Accepting this notation, consider the following individual cases.

1) $x \in(0, a)$. In this case we have

$$
\begin{gathered}
I_{n}^{(1)}+I_{n}^{(3)}=\int_{0}^{a}[f(t)+f(-t)] \bar{\vartheta}_{n}^{+}(t) d t\left[\Phi_{n}(x)+\Phi_{n}(-x)\right]= \\
=\int_{0}^{a}[f(t)+f(-t)] \bar{\vartheta}_{n}^{+}(t) d t\left[\varphi_{n}(x)+\psi_{n}(x)\right]= \\
=\int_{0}^{a}[f(t)+f(-t)] \bar{\vartheta}_{n}^{+}(t) d t x_{n}^{+}(x),
\end{gathered}
$$




$$
\begin{gathered}
I_{n}^{(2)}-I_{n}^{(4)}=\int_{0}^{a}[f(t)-f(-t)] \bar{\vartheta}_{n}^{-}(t) d t\left[\Phi_{n}(x)-\Phi_{n}(-x)\right]= \\
\int_{0}^{a}[f(t)-f(-t)] \bar{\vartheta}_{n}^{-}(t) d t x_{n}^{-}(x) .
\end{gathered}
$$

Considering these expressions, we obtain

$$
\begin{gathered}
I_{m}^{(0, a)}=\| \sum_{n=1}^{m} \frac{1}{2}\left(I_{n}^{(1)}+I_{n}^{(3)}\right)-\frac{f(x)+f(-x)}{2}+ \\
\sum_{n=1}^{m} \frac{1}{2}\left(I_{n}^{(2)}-I_{n}^{(4)}\right)-\frac{f(x)-f(-x)}{2} \|_{L^{p}(0, a)} \leq \\
\leq\left\|\sum_{n=1}^{m} \frac{1}{2}\left(I_{n}^{(1)}+I_{n}^{(3)}\right)-f^{+}(x)\right\|_{L^{p}(0, a)}+ \\
\left\|\sum_{n=1}^{m} \frac{1}{2}\left(I_{n}^{(1)}+I_{n}^{(3)}\right)-f^{+}(x)\right\|_{L^{p}(0, a)},
\end{gathered}
$$

where $f^{ \pm}(x)=\frac{f(x) \pm f(-x)}{2}$. Drawing attention to the relations

$$
\begin{aligned}
& \sum_{n=1}^{m} \frac{1}{2}\left(I_{n}^{(1)}+I_{n}^{(3)}\right)=\sum_{n=1}^{m} \int_{0}^{a} f^{+}(t) \bar{\vartheta}_{n}^{+}(t) d t x_{n}^{+}(x), \\
& \sum_{n=1}^{m} \frac{1}{2}\left(I_{n}^{(2)}-I_{n}^{(4)}\right)=\sum_{n=1}^{m} \int_{0}^{a} f^{-}(t) \bar{\vartheta}_{n}^{-}(t) d t x_{n}^{-}(x),
\end{aligned}
$$

from the previous inequality follows $I_{m}^{(0, a)} \rightarrow 0, m \rightarrow \infty$.

2) $x \in(-a, 0)$. In this case it holds

$$
\begin{gathered}
I_{n}^{(1)}+I_{n}^{(3)}=2 \int_{0}^{a} f^{+}(t) \bar{\vartheta}_{n}^{+}(t) d t\left[\psi_{n}(x)+\varphi_{n}(-x)\right]= \\
2 \int_{0}^{a} f^{+}(t) \bar{\vartheta}_{n}^{+}(t) d t x_{n}^{+}(-x), \\
I_{n}^{(2)}-I_{n}^{(4)}=2 \int_{0}^{a} f^{-}(t) \bar{\vartheta}_{n}^{-}(t) d t\left[\psi_{n}(-x)-\varphi_{n}(-x)\right]= \\
-2 \int_{0}^{a} f^{-}(t) \bar{\vartheta}_{n}^{-}(t) d t x_{n}^{-}(-x) .
\end{gathered}
$$

Similarly to the previous case we have

$$
\begin{gathered}
I_{m}^{(-a ; 0)}=\| \sum_{n=1}^{m} \frac{1}{2}\left(I_{n}^{(1)}+I_{n}^{(3)}\right)-f^{+}(x)+ \\
+\sum_{n=1}^{m} \frac{1}{2}\left(I_{n}^{(2)}-I_{n}^{(4)}\right)-f^{-}(x) \|_{L^{p}(-a, 0)} \\
\leq\left\|\sum_{n=1}^{m} \int_{0}^{a} f^{+}(t) \bar{\vartheta}_{n}^{+}(t) d t x_{n}^{+}(x)-f^{+}(x)\right\|_{L^{p}(0, a)}+ \\
\left\|-\sum_{n=1}^{m} \int_{0}^{a} f^{-}(t) \bar{\vartheta}_{n}^{-}(t) d t x_{n}^{-}(x)-f^{-}(-x)\right\|_{L^{p}(0, a)},
\end{gathered}
$$

$m \rightarrow \infty$, so , $f^{-}(-x)=-f^{-}(x)$. Then from here it follows $I_{m}^{(-a, a)} \rightarrow 0, m \rightarrow \infty$, i.e.

$f(x)=\sum_{n=1}^{\infty}\left(\left\langle f ; h_{n}^{+}\right\rangle_{(-a, a)} \Phi_{n}(x)+\left\langle f ; h_{n}^{-}\right\rangle_{(-a, a)} \Psi_{n}(x)\right)$,

where

$$
\langle f ; g\rangle_{(c, d)}=\int_{c}^{d} f(t) \overline{g(t)} d t .
$$

For simplicity we will denote $g(f)=\langle f ; g\rangle_{(c, d)}$. Take $\forall f \in L_{p}(-a, a)$. We have

$$
\begin{aligned}
& h_{n}^{ \pm}(f)= \frac{1}{2}\left(\int_{-a}^{a} f(t) \bar{\Omega}_{n}^{+}(t) d t \pm \int_{-a}^{a} f(t) \bar{\Omega}_{n}^{-}(t) d t\right)= \\
& \frac{1}{2}\left(\int_{0}^{a} f(t) \bar{\vartheta}_{n}^{+}(t) d t+\int_{-a}^{0} f(t) \bar{\vartheta}_{n}^{+}(-t) d t \pm\right. \\
& \int_{0}^{a} f(t) \bar{\vartheta}_{n}^{-}(t) d t \mp \int_{-a}^{0} f(t) \bar{\vartheta}_{n}^{-}(-t) d t= \\
&=\int_{0}^{a} f^{+}(t) \bar{\vartheta}_{n}^{+}(t) d t \pm \int_{0}^{a} f^{-}(t) \bar{\vartheta}_{n}^{-}(t) d t=\vartheta_{n}^{+}\left(f^{+}\right) \\
& \pm \vartheta_{n}^{-}\left(f^{-}\right), \forall n \in N .
\end{aligned}
$$

Thus

$$
h_{n}^{ \pm}(f)=\vartheta_{n}^{+}\left(f^{+}\right) \pm \vartheta_{n}^{-}\left(f^{-}\right), \forall n \in N \text {. }
$$

Hence

$$
\begin{array}{r}
\left\|\left\{h_{n}^{ \pm}(f)\right\}\right\|_{\mathrm{K}} \leq\left\|\left\{\vartheta_{n}^{+}\left(f^{+}\right)\right\}\right\|_{\mathrm{K}}+\left\|\left\{\vartheta_{n}^{-}\left(f^{-}\right)\right\}\right\|_{\mathrm{K}} \leq \\
B^{+}\left\|f^{+}\right\|_{L_{p}(0, a)}+B^{-}\left\|f^{-}\right\|_{L_{p}(0, a)},
\end{array}
$$

where $B^{ \pm}$is the upper frame bound with respect to the system $\vec{x}^{ \pm}$. We have

$$
\begin{gathered}
\|f\|_{L^{p)}(-a, a)}=\left\|f^{+}+f^{-}\right\|_{L^{p)}(-a, a)} \leq \\
\left\|f^{+}\right\|_{L^{p)}(-a, a)}+\left\|f^{-}\right\|_{L^{p)}(-a, a)} .
\end{gathered}
$$

Considering that $\left\|f^{ \pm}\right\|$is an even function, we have

$$
\begin{aligned}
& \left\|f^{ \pm}\right\|_{L^{p}(-a, a)}=\sup _{0<\varepsilon<p-1}\left(\frac{\varepsilon}{2 a} \int_{-a}^{a}\left|f^{ \pm}(t)\right|^{p-\varepsilon} d t\right)^{\frac{1}{p-\varepsilon}}= \\
& =\sup _{0<\varepsilon<p-1}\left(\frac{\varepsilon}{a} \int_{0}^{a}\left|f^{ \pm}(t)\right|^{p-\varepsilon} d t\right)^{\frac{1}{p-\varepsilon}}=\left\|f^{ \pm}\right\|_{L^{p}(0, a)} .
\end{aligned}
$$

Consequently

$$
\|f\|_{L^{p)}(-a, a)} \leq\left\|f^{+}\right\|_{L^{p}(0, a)}+\left\|f^{-}\right\|_{L^{p}(0, a)} .
$$

On the other hand we have 


$$
\begin{aligned}
& \left\|f^{ \pm}\right\|_{L^{p}(0, a)}=\left\|f^{ \pm}\right\|_{L^{p}(-a, a)}=\left\|\frac{f(t) \pm f(-t)}{2}\right\|_{L^{p}(-a, a)} \leq \\
& \leq \frac{1}{2}\left(\|f(t)\|_{L^{p}(-a, a)}+\|f(-t)\|_{L^{p)}(-a, a)}\right)=\|f\|_{L^{p}(-a, a)},
\end{aligned}
$$

Since

$$
\|f(t)\|_{L^{p}(-a, a)}=\|f(-t)\|_{L^{p)}(-a, a)} .
$$

As a result we obtain

$$
\begin{gathered}
\left\|f^{+}\right\|_{L^{p}(0, a)}+\left\|f^{-}\right\|_{L^{p)}(0, a)}=\left\|f^{+}\right\|_{L^{p}(-a, a)} \\
+\left\|f^{-}\right\|_{L^{p}(-a, a)} \leq 2\|f\|_{L^{p}(-a, a)} .
\end{gathered}
$$

Thus, the following inequality

$$
\begin{aligned}
\|f\|_{L^{p)}(-a, a)} & \leq\left\|f^{+}\right\|_{L^{p)}(0, a)}+\left\|f^{-}\right\|_{L^{p)}(0, a)} \\
& \leq 2\|f\|_{L^{p)}(-a, a)},
\end{aligned}
$$

holds. Taking into account the above relation from (3.3) we obtain

$$
\left\|\left\{h_{n}^{+}(f)\right\}\right\|_{\mathrm{K}}+\left\|\left\{h_{n}^{-}(f)\right\}\right\|_{\mathrm{K}} \leq 2 B\|f\|_{L^{p}(-a, a)},
$$

where $B=\max \left\{B^{+} ; B^{-}\right\}$. On the other hand from (3.2) it follows

$$
\vartheta_{n}^{ \pm}\left(f^{ \pm}\right)=\frac{1}{2}\left(h_{n}^{+}(f) \pm h_{n}^{-}(f)\right), \forall n \in N .
$$

Consequently

$$
\begin{aligned}
& \left\|\left\{h_{n}^{+}(f)\right\}\right\|_{\mathrm{K}}+\left\|\left\{h_{n}^{-}(f)\right\}\right\|_{\mathrm{K}} \geq \|\left\{\vartheta _ { n } ^ { + } ( f ^ { + } ) \| _ { \mathrm { K } } + \| \left\{\vartheta_{n}^{-}\left(f^{-}\right) \|_{\mathrm{K}} \geq\right.\right. \\
& \geq A^{+}\left\|f^{+}\right\|_{L^{p}(0, a)}+A^{-}\left\|f^{-}\right\|_{L^{p}(0, a)} \geq A\|f\|_{L^{p}(-a, a)} \\
& \quad A\left(\left\|f^{+}\right\|_{L^{p}(0, a)}+\left\|f^{-}\right\|_{L^{p}(0, a)}\right) \geq, \\
& \text { where } A=\min \left\{A^{+} ; A^{-}\right\} . \\
& \text {Theorem is proved. }
\end{aligned}
$$

\section{References:}

[1] Young R. An introduction to nonharmonic Fourier series, Academic Press, New York, 1980

[2] Daubechies I. Ten lectures on wavelets, SIAM, Philadelphia. 1992.

[3] Christensen O. A short introduction to frames, Gabor systems, and wavelet systems, Azerb. Journal of Mathematics. 4(1), 2014, pp.25-39

[4] Christensen O. An Introduction to Frames and Riesz Base, Birkhauser Boston, 2002.

[5] Christensen O. Frames and bases. An introductory course, Birkhauser, Boston. 2008.

[6] Christensen O., Stoeva D.T. $p$-frames in separable Banach spaces, Advances in Computational Mathematics. 18 (2003), 17 126.
[7] Chui Ch. Wavelets: a tutorial in theory and applications, Academic Press, Boston. 1992, $724 \mathrm{p}$.

[8] Dremin I.M., Ivanov O.V., Nechitailo V.A. Wavelets and their uses, Usp. phisics nauk, 2001, 171 (5), 465-501.

[9] Duffin R. J., Schaeffer A. C. A class of nonharmonic Fourier series, Trans. Amer.Math. Soc. 72(1952), $341-366$.

[10] Bilalov B. T. On basicity of system $\{\exp (i \sigma n x) \sin n x\}$ and exponents with a shift, Dokl. RAN, 1995, vol. 345, No.2, pp. 151-152

[11] Bilalov B. T. Basis properties of some exponential systems and powers with shift, Russian Acad. Sci., Dokl. Math., v.49 (1994), No. 1, pp.107-112

[12] Bilalov B. T. A system of exponential functions with shift and the Kostyuchenko problem, Sibirsk. Mat. Zh., 50:2 (2009), pp. 279-288

[13] Bilalov B. T. On solution of the Kostyuchenko problem, Sibirsk. Mat. Zh., 53:3 (2012), pp. 509-526

[14] Bilalov B. T. The basis properties of some systems of exponential functions, cosines, and sines, Sibirsk. Mat. Zh., 45:2 (2004), pp. 264273

[15] Bilalov B. T. The basis properties of power systems in $L_{p}$, Sibirsk. Mat. Zh., 47:1 (2006), pp. 25-36

[16] Bilalov B.T., Guseinov Z.G. $K$-Bessel and $K$ Hilbert systems and $K$-bases. Dokl. Mathematics, vol. 80, no. 3, 2009, pp. 826-828

[17] Bilalov B.T., Guseinov Z.G. ${ }^{p}$-Bessel and $p_{\text {- }}$

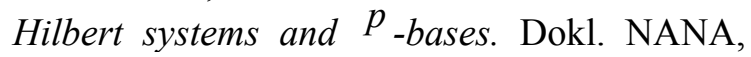
vol. LXIV, no. 3, 2008, pp. 3-8

[18] Feichtinger H.G. Gröchenig K.H. Banach Spaces Related to Integrable Group Representations and Their Atomic Decompositions. Part I, J. Funct. Anal., 86 (1989), pp. 307-340

[19] Feichtinger H.G. Gröchenig K.H. Banach Spaces Related to Integrable Group Representations and Their Atomic Decompositions. Part II, Monatshefte für Mathematik, 108(1989), pp. 129-148

[20] Gröchenig K.H. Describing functions: frames versus atomic decompositions, Monatch. Math., 112(1991), pp. 1-41

[21] Aldroubi A., Sun Q., Tang W. P-frames and shift invariant subspaces of $L^{p}$, J. Fourier Anal. Appl., 7, No 1 (2001), pp. 1-22 
[22] Xianling F., Dun Z. On the spaces $L^{p(x)}(\Omega)$ and $W^{m, p(x)}(\Omega)$, J. Math. Anal. Appl. 2001, v. 263, № , pp. 424-446

[23] Sharapudinov I.I. On the topology of the space $L^{p(\cdot)}(0 ; 1)$. Mat. zametki, 1979, v. 26, № 4, pp. 613-632 (in Russian)

[24] Zorko C.T. Morrey spaces, Proc. Amer. Math. Soc. 1986, v. 98, № 4, pp. 586-592.

[25] Morrey C.B., On the solutions of quasi-linear elliptic partial differential equations, Trans. Amer. Math. Soc. 1938, v. 43, № 4, pp. 207226.

[26] Cruz-Uribe D. V., Fiorenza A. Variable Lebesgue spaces: Foundations and harmonic analysis. Springer-Verl., 2013.

[27] Adams D. R. Morrey spaces. Springer Intern. Publ.: Switzerland, 2016.

[28] N. Samko, Weight Hardy and singular operators in Morrey spaces, J. Math. Anal. Appl. 35 (2009), № 1, pp. 183-188.

[29] V. Kokilashvili. Boundedness criteria for singular integrals in weighted grand Lebesgue spaces. J. Math. Sci., 170, 2010, pp. 20-33.

[30] R.E.Castillo, H.Rafeiro, An Introductory Course in Lebesgue Spaces, Springer International Publishing Switzerland, 2016.

[31] A. Fiorenza, G. E. Karadzhov, Grand and small Lebesgue spaces and their analogs. Z. Anal. Anwend. 23(4) (2004), pp. 657-681.

[32] Bilalov B.T., Gasymov T.B., Guliyeva A.A. On the solvability of the Riemann boundary value problem in Morrey-Hardy classes, Turkish Journal of Mathematics 40 (5), 2016, pp. 10851101.

[33] Sharapudinov I. I. On direct and inverse theorems of approximation theory in variable Lebesgue and Sobolev spaces, Azerbaijan J. Math. 2014. v. 4, №1, pp. 55-72.

[34] Israfilov D. M., Tozman N. P. Approximation by polynomials in Morrey-Smirnov classes, East J. Approx. 2008. V. 14, № 3, pp. 255-269.

[35] Israfilov D. M., Tozman N. P. Approximation in Morrey-Smirnov classes, Azerbaijan J. Math. 2011. v. 1, № 1. pp. 99-113.

[36] Bilalov B. T., Guseynov Z.G. Basicity of a system of exponents with a piece-wise linear phase in variable spaces, Mediterr. J. Math., 2012, v. 9, № 3, pp. 487-498.

[37] B.T.Bilalov, A.A.Quliyeva, On basicity of exponential systems in Morrey-type spaces, International Journal of mathematics, v. 25, 6, 2014, 1450054 (1-10).

[38] Iwaniec T., Sbordone C. On the integrability of the Jacobian under minimal hypotheses, Arch.
Ration. Mech. Anal., 119(2), 1992, pp. 129143 